\section{Continuo ascenso de la prevalencia de diabetes mellitus en mujeres chilenas en edad fértil: proyección 2020-2039 de malformaciones congénitas por diabetes pregestacional}

\author{
PABLO OLMOS COELHO ${ }^{1,2,3}$, GISELLA BORZONE TASSARA ${ }^{4}$, \\ ANDRÉS POBLETE LIZANA ${ }^{1}$, ENRIQUE OYARZÚN EBENSPERGER ${ }^{5}$
}

\section{Cost-effectiveness of a diabetes detection program in childbearing women to prevent malformations}

Background: In Chile, in the next 20 years there will be an average number of 537,100 women of childbearing age with type 2 diabetes (T2DM). Half of these women, not knowing their diagnosis of diabetes will become pregnant with elevated glycosylated hemoglobin levels, increasing to 25\% the risk of having offspring with congenital malformations. Aim: To calculate the cost-effectiveness of a diabetes detection strategy in women of childbearing age for the prevention of congenital malformations in the period 2020-2039. Material and Methods: The Markov stochastic model was used in two scenarios, namely A: No intervention and B: Intervention, detecting T2DM in Chilean women aged 15-49 years. We calculated the years of life lost due to premature death (YLL), years of life lost adjusted for disability (DALY) and total costs using American dollars (US\$) of 2017, with a discount rate of 5\%. Results: In scenario A: 63,330 children with malformations would be born, YLLs and DALYs would be 800,750 and 1,544,775 respectively, and US\$10,194,298,011 would be expended. In scenario B, these figures would be reduced in approximately $70 \%$ to 19,025 children born with malformations, YLLS and DALYs would be 40,539 and 464,033 respectively and US\$3,354,321,968 would be expended. The costs of prevention would be US\$ 6,590.94/malformation avoided, US\$ 521.33/YLL avoided, and US\$ 270.24/ $D A L Y$ avoided. Conclusions: In the context of an increasing prevalence of diabetes in women of childbearing age, our results suggest that the early detection of diabetes in Chilean women between 15 and 49 years of age, should be cost-effective to avoid an increase in congenital malformations for the next 20 years.

(Rev Med Chile 2020; 148: 1171-1182)

Key words: Congenital Abnormalities; Diabetes Mellitus, Type 2; Markov Chains; Prevalence; Prevention \& Control.
'Departamento de Obstetricia-

Ginecología, Facultad de

Medicina, Pontificia Universidad

Católica de Chile. Santiago, Chile.

${ }^{2}$ Ingeniería Biomédica. Facultad

de Ingeniería, Pontificia

Universidad Católica de Chile.

Santiago, Chile.

${ }^{3}$ Departamento de Nutrición-

Diabetes-Metabolismo,

Facultad de Medicina, Pontificia

Universidad Católica de Chile.

Santiago, Chile.

${ }^{4}$ Departamento de Enfermedades

Respiratorias, Facultad de

Medicina, Pontificia Universidad

Católica de Chile. Santiago, Chile.

${ }^{5}$ Departamento de Obstetricia

y Ginecología, Facultad de

Medicina Universidad de los

Andes, Clínica Universidad de Los

Andes. Santiago, Chile.

Recibido el 17 de octubre de 2019, aceptado el 21 de julio de 2020.

Correspondencia a:

Dr. Pablo Olmos

Facultad de Medicina, Pontificia

Universidad Católica de Chile.

Avenida Libertador Bernardo O'Higgins 340. Santiago, Chile. polmos@med.puc.cl

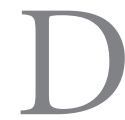
urante el siglo XX, en Chile, la mortalidad infantil se redujo de 342 a 8,9 por cada 1.000 nacidos vivos. En los primeros 80 años del siglo, esa reducción ocurrió a expensas del control de la desnutrición, diarrea y enfermedades transmisibles ${ }^{1}$, pero el ritmo de disminución fue menor después de 1980, en la medida que las causas predominantes pasaron a ser las complicaciones maternas del embarazo y las malformaciones congénitas ${ }^{1,2}$. 
Precisamente, una de las complicaciones maternas que está aumentando actualmente es la diabetes mellitus pregestacional (DMPG), término que se refiere a mujeres con diabetes mellitus tipo 1 (DMT1), diabetes mellitus tipo 2 (DMT2) o diabetes mellitus secundaria, presentes antes del embarazo. Se excluye, entonces, diabetes gestacional. Además de otras complicaciones, la DMPG se asocia a una incidencia elevada de malformaciones congénitas ${ }^{3}$.

La DMT2 -antes limitada a adultos mayoresestá aumentando en todo el mundo en personas jóvenes, lo que incluye a las mujeres en edad fértil (MEF) (15 a 49 años) ${ }^{4-6}$, quienes, en caso de embarazo, tienen un riesgo de malformaciones congénitas exponencialmente proporcional al nivel de hiperglicemia al momento de la concepción que se muestra en Figura 1 como regresión exponencial a partir de cuatro publicaciones ${ }^{7-10}$. Estas malformaciones pueden reducirse drásticamente mediante un estricto control de la glicemia materna, asegurando una hemoglobina glicosilada (HbAlc) $<6,5 \%$ durante 6 meses antes de la concepción y también durante todo el embarazo $^{11}$. No hay estudios con cifras de HbAlc al momento del diagnóstico de DM en mujeres en edad fértil. En jóvenes entre 15 y 19 años con DMT2 no diagnosticada se han encontrado valores de $\mathrm{HbA} 1 \mathrm{c}$ promedio de $11,5 \%{ }^{12}$, niveles que, si están presentes al momento de la concepción, conllevan un riesgo de malformaciones de hasta $25 \%$ (Figura 1).

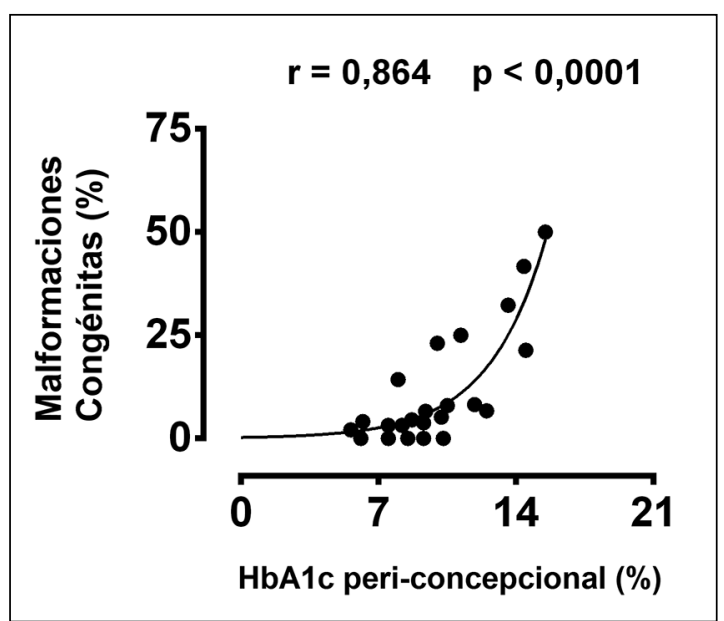

Figura 1. Aumento exponencial de malformaciones congénitas a medida que aumenta la $\mathrm{HbA} 1 \mathrm{c}$ al momento de la concepción?.
Entre 2003 y 2010, la prevalencia de diabetes mellitus entre mujeres chilenas de 25 a 44 años subió 47 veces, lo que nos permitió estimar en 2018 que, de no tomarse medidas preventivas, en los siguientes 20 años nacerían 12.163 infantes con malformaciones congénitas ${ }^{13}$. En 2019, el Ministerio de Salud publicó resultados preliminares de la Encuesta Nacional de Salud 2016-2017, los que revelan que la prevalencia de diabetes mellitus en mujeres en edad fértil (MEF-DM), definida como 15-49 años, continúa aumentando en Chile $^{14}$. En este contexto, nuestros objetivos fueron los siguientes: (a) Estimar número de MEF-DM, tanto en la actualidad (2019), como para los siguientes 20 años. (b) Con un modelo de Markov, calcular el número de infantes con malformaciones que nacerán de MEF-DM en los próximos 20 años. (c) Estudiar la relación costo-efectividad de una estrategia de detección de diabetes mellitus en MEF para la prevención de malformaciones congénitas.

\section{Métodos}

Compatibilizamos los resultados publicados en estadísticas de población, provenientes del Instituto Nacional de Estadísticas (INE), con las publicaciones del Ministerio de Salud (MINSAL) en la Tabla $1^{15,16}$.

Como los datos del censo 2012 no deben usar$\mathrm{se}^{17}$, calculamos para ese año los valores medios entre los de los censos de $2003^{16}$ y $2017^{18,19}$ (Tabla 2).

Debido a las diferencias en los rangos de edad publicados por el Ministerio de Salud por un lado, y por el Instituto Nacional de Estadísticas, por otro, para el año 2017 (Tabla 3) la prevalencia de diabetes mellitus publicada para el rango-MINSAL " $45-64$ " $(18,3 \%)^{11}$ se aplicó a la población en el rango-INE "45-49"18. Cada una de las cifras de MEF-DM debe considerarse como la suma de dos subgrupos: aquellas con diabetes mellitus previamente diagnosticadas y en tratamiento (MEFDM-Dx, 53,08\%), y aquellas no diagnosticadas o diagnosticadas pero sin tratamiento (MEF-DMnoDx, $46,92 \%)^{20}$.

\section{Justificación del uso del modelo de Markov}

(i) En la situación actual (escenario "A"), sin detección universal de diabetes mellitus (DM) en MEF, utilizamos un modelo de Markov 
de seis estados para calcular la incidencia de malformaciones para los próximos 20 años en MEF-DM chilenas, de los cuales 53,08\% serán MEF-DM-Dx y 46,92\% serán MEFDM-noDx. El acrónimo de estas últimas significa "MEF-diabéticas-no diagnosticadas o que saben su diagnóstico pero no se tratan".

(ii) En una situación ideal (escenario "B"), con detección universal de DM en MEF, utilizamos un modelo de Markov de seis estados para calcular la incidencia de recién nacidos con malformaciones en Chile durante los próximos 20 años, en MEF-DM chilenas, de las cuales $100 \%$ será MEF-DM-Dx.

(iii) Combinando los resultados de (i) y (ii), calculamos los beneficios de la detección universal de DM en MEF en Chile, en los próximos 20 años, medidos por: (a) Reducción en el número de recién nacidos con malformaciones; (b) reducción en años de vida perdidos y (c) reducción de los costos totales de por vida (US\$, año 2017).

\section{Parámetros y probabilidades}

Entre los parámetros generales (Tablas 1 y 4), hay tres que deben explicarse en detalle: (i) Consideramos la tasa global de fertilidad en Chile " $f$ ", 1,8 nacimientos vivos/mujer ${ }^{21}$. (ii) En Chile 2010, la probabilidad global de malformaciones congénitas $(\mathrm{gpm})$ entre los nacidos vivos fue 3,9\% $(g p m=0,039)^{22}$. La proporción " $e$ " son las MEF-DM-Dx que han sido diagnosticadas y están en tratamiento, ya sea este farmacológico o no farmacológico ( $e=0,5308$ para el escenario A $)^{20}$. El 46,92\% restante, correspondiente a las MEF-DM-noDx, no está recibiendo tratamiento alguno. Para efectos del modelo de Markov, los autores consideramos la última cifra de $46,92 \%$, como "no diagnosticadas o no tratadas".

\section{Análisis}

El modelo de Markov fue construido de acuerdo al método de Drummond et al. ${ }^{23}$. Brevemente, dibujamos un diagrama de influencia para escenario A (Figura 2). Las probabilidades de transición mostradas en Tabla 4 fueron introducidas en una matriz de transición (" $P$ ") de 6 por 6 celdas (ecuación 6).

Para comprender el modelo de Markov, expondremos lo que sucede al pasar del "estado 1" (no embarazada, sin malformación) al "estado 2" (primer embarazo, con malformación). Vea el diagrama de influencia para el escenario " $A$ " (Figura 4). Las mujeres en edad fértil con DM (A-MEF-DM) en el estado 1 tienen una probabilidad anual de $\theta_{\text {a12 }}=0,01242$ de primer embarazo con malformación congénita, con lo cual pasarían al estado 2. La ecuación 1 ilustra el cálculo de $\theta_{\mathrm{a} 12}$ :

$$
\begin{array}{r}
\theta_{\mathrm{a} 12}=(\text { A.MEF.DM.DX.12 })+(\text { A.MEF.DMNODX.12) } \\
\theta_{\mathrm{a} 12}=(0,0018631)+(0,010557) \\
\theta_{\mathrm{a} 12}=0,01242
\end{array}
$$

Ecuación 1

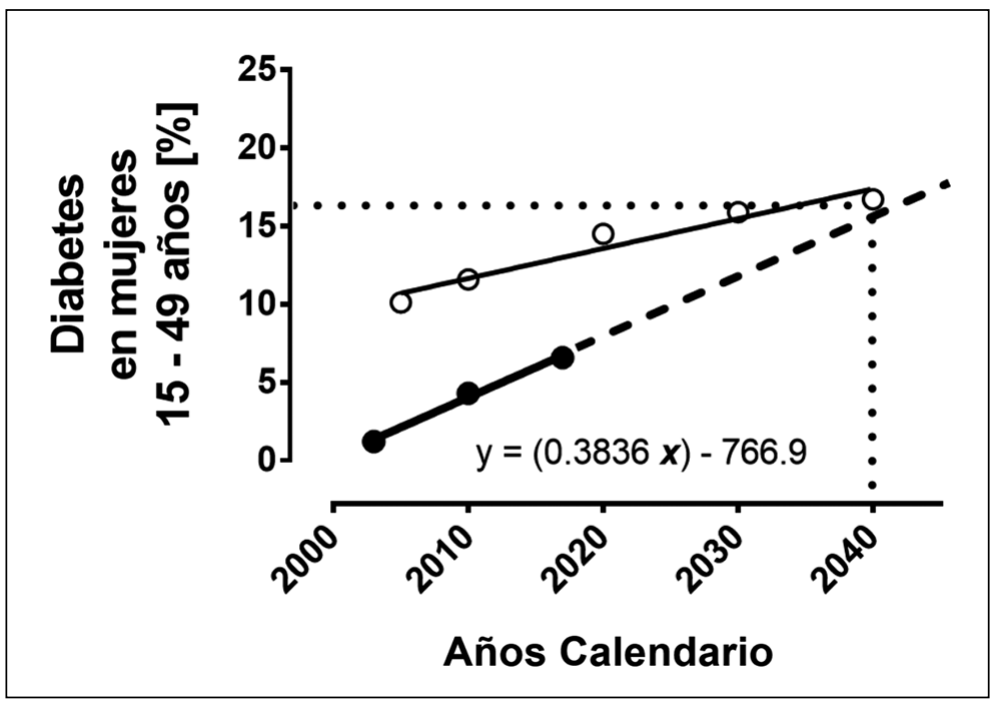

Figura 2. Prevalencia de diabetes mellitus en mujeres en edad fértil (15-49 años), calculada en Chile entre 2003 y $2017(\bullet)$ y extrapolada hasta el año 2040 (----). La línea continua más delgada muestra la prevalencia de diabetes mellitus en mujeres de 20 a 44 años en U.S.A. para el mismo período (o) ${ }^{26}$. Las líneas vertical y horizontal de puntos (.....) muestran que para el año 2040, las prevalencias de DM en Chile y en U.S.A. convergerían a una cifra cercana a $16 \%$. 
Tabla 1. Estimación de parámetros generales, intermediarios y definitivos, para los escenarios " $A$ " $y$ " $B$ "

\begin{tabular}{|c|c|c|}
\hline Definición & Nombre & Valor \\
\hline $\begin{array}{l}\text { En escenario A, probabilidad anual, en A-MEF-DM (con diabetes diagnosticada } \\
\text { o no), de tener malformación en } 1^{\circ} \text { embarazo }\end{array}$ & $\theta_{\mathrm{a} 12}$ & 0,0124201 \\
\hline $\begin{array}{l}\text { En escenario B, probabilidad anual, en B-MEF-DM, de tener malformación en } 1^{\circ} \\
\text { embarazo }\end{array}$ & $\theta_{\mathrm{b} 12}$ & 0,00351 \\
\hline $\begin{array}{l}\text { En escenario A, probabilidad anual, en A-MEF-DM (con diabetes diagnosticada } \\
\text { o no), de no tener malformación en } 1^{\circ} \text { embarazo }\end{array}$ & $\theta_{a 13}$ & 0,077579 \\
\hline $\begin{array}{l}\text { En escenario A, probabilidad anual, en A-MEF-DM (con diabetes diagnosticada } \\
\text { o no), de NO embarazarse }\end{array}$ & $\theta_{a 11}$ & 0,910 \\
\hline $\begin{array}{l}\text { En escenario B, probabilidad anual, en B-MEF-DM (con diabetes diagnosticada o } \\
\text { no), de no tener malformación en } 1^{\circ} \text { embarazo }\end{array}$ & $\theta_{\mathrm{b}_{13}}$ & 0,08649 \\
\hline En escenario B, probabilidad anual, en B-MEF-DM, de NO embarazarse & $\theta_{\mathrm{b} 11}$ & 0,910 \\
\hline $\begin{array}{l}\text { En escenario A, probabilidad anual, en A-MEF-DM, quien ya tuvo malformación } \\
\text { en } 1^{\circ} \text { embarazo, de tener de nuevo malformación en el } 2^{\circ} \text { embarazo }\end{array}$ & $\theta_{\mathrm{a} 24}$ & 0,00351 \\
\hline $\begin{array}{l}\text { En escenario } B \text {, probabilidad anual, en B-MEF-DM, quien ya tuvo malformación } \\
\text { en } 1^{\circ} \text { embarazo, de tener de nuevo malformación en el } 2^{\circ} \text { embarazo }\end{array}$ & $\theta_{\mathrm{b} 24}$ & 0,00351 \\
\hline $\begin{array}{l}\text { En escenario A, probabilidad anual, en A-MEF-DM, quien no tuvo malformación } \\
\text { en } 1^{\circ} \text { embarazo, de tener una malformación en el } 2^{\circ} \text { embarazo }\end{array}$ & $\theta_{\text {аз }}$ & 0,00351 \\
\hline $\begin{array}{l}\text { En escenario } B \text {, probabilidad anual, en B-MEF-DM, quien no tuvo malformación } \\
\text { en } 1^{\circ} \text { embarazo, de tener una malformación en el } 2^{\circ} \text { embarazo }\end{array}$ & $\theta_{\mathrm{b} 36}$ & 0,00351 \\
\hline $\begin{array}{l}\text { En escenario A, probabilidad anual, en A-MEF-DM, quien no tuvo malformación } \\
\text { en } 1^{\circ} \text { embarazo, de no tener una malformación en el } 2^{\circ} \text { embarazo }\end{array}$ & $\theta_{\text {a35 }}$ & 0,99649 \\
\hline $\begin{array}{l}\text { En escenario B, probabilidad anual, en B-MEF-DM, quien no tuvo malformación } \\
\text { en } 1^{\circ} \text { embarazo, de no tener una malformación en el } 2^{\circ} \text { embarazo }\end{array}$ & $\theta_{\mathrm{b} 35}$ & 0,99649 \\
\hline $\begin{array}{l}\text { En escenario A, probabilidad anual, en A-MEF-DM, quien ya tuvo malformación } \\
\text { en } 1^{\circ} \text { embarazo, de no tener una malformación en el } 2^{\circ} \text { embarazo }\end{array}$ & $\theta_{\mathrm{a} 26}$ & 0,99649 \\
\hline $\begin{array}{l}\text { En escenario } B \text {, probabilidad anual, en B-MEF-DM, quien ya tuvo malformación } \\
\text { en } 1^{\circ} \text { embarazo, de no tener una malformación en el } 2^{\circ} \text { embarazo }\end{array}$ & $\theta_{\mathrm{b} 26}$ & 0,99649 \\
\hline $\begin{array}{l}\text { En el escenario A, probabilidad para A-MEF-DM en estados } 4,5 \text { y } 6 \text {, de } \\
\text { permanecer en estos estados absorbentes }\end{array}$ & $\theta_{a 44}, \theta_{a 55}, \theta_{a 66}$ & 1,0 \\
\hline $\begin{array}{l}\text { En el escenario B, probabilidad para B-MEF-DM en estados } 4,5 \text { y } 6 \text {, de } \\
\text { permanecer en estos estados absorbentes }\end{array}$ & $\theta_{\mathrm{b} 44}, \theta_{\mathrm{b} 55}, \theta_{\mathrm{b} 66}$ & 1,0 \\
\hline
\end{tabular}

El valor de $\theta_{\text {a12 }}$ es la suma de dos probabilidades de pasar del estado 1 al 2: A.MEF.DM.DX.12 es la probabilidad en aquel 53,08\% de A-MEF-DM diagnosticadas y tratadas, y A.MEF.DM.NODX.12 es esa probabilidad en aquel 46,92\% de las A-MEFDM que no lo están.

Para determinar el efecto del tratamiento, vale decir, el escenario $B$, se efectúa un procedimiento similar al anterior, pero con probabilidades de malformación mucho menores, similares a los de la población general.

El vector de ciclo cero (frecuencias iniciales, Tabla 6) fue multiplicado 20 veces en la matriz $P$ (representando 20 años), originando un vector resultante (vector $=$ matriz de una sola fila), que contiene las frecuencias proyectadas al año 2039, de los cuales solo nos interesan el estado 4 (2 malformaciones en 20 años) y el estado 6 ( 1 malfor- 
mación en 20 años). Para el escenario B hicimos un procedimiento similar (Figura 3 y ecuación 7 ).

\section{Costos}

Los costos totales de por vida incluyeron dos tipos. (i) Costos de por vida, que es el dinero gastado por pacientes, familias e instituciones de salud durante toda la expectativa de vida de infantes nacidos con malformaciones. Este es el único tipo de costos en el escenario modelo $\mathrm{A}$, y es uno de los costos del escenario B. (ii) En el escenario B, tenemos, además, costos de detección y paciente nuevo, en que se incurriría en los próximos 20 años para detectar y tratar las pacientes diagnosticadas con DMT2.

Cálculo de costos de por vida: Tabla 5 muestra los costos de por vida y los años de vida perdidos por muerte prematura (YLL, por su nombre en inglés: Years of Life Lost), lo que resulta en una cifra media (no descontada) de US\$ 8.502,58 por niño/a por año de expectativa de vida. En cada uno de los 20 años del período 2020-2039 $\left(\mathrm{P}_{1}, \mathrm{P}_{2}, \ldots . \mathrm{P}_{\mathrm{n}}\right)$, el costo futuro de las malformaciones se calculó con una tasa de descuento de $5 \%$. Finalmente, los 20 valores de $\mathrm{P}_{\mathrm{n}}$ se sumaron, como se muestra en la parte inferior de la Tabla 6. Como fue mencionado en el párrafo anterior, en el escenario A, este valor de $\mathrm{P}$ fue igual al costo total de por vida.

El modelo en escenario B (Tabla 7), en cambio, además de su costo de por vida, tiene el costo de detección y tratamiento de paciente nuevo.

El método de detección propuesto es la medición de $\mathrm{HbAlc}$ en sangre venosa en todos las MEF que asistan por cualquier razón a un centro ambulatorio de obstetricia y ginecología ${ }^{24-26}$. Un valor de $\mathrm{HbAlc}$ igual o superior a $6,5 \%$ se considera sugerente de DMT2 ${ }^{25,26}$.

Este costo de detección de DMT2 por HbA1c en personas no preseleccionadas se ha establecido en US\$433,39 actuales, lo que incluye la confirmación del diagnóstico y los costos asociados de personal y laboratorio ${ }^{24}$. A este costo de detección, agregamos el costo del tratamiento, de US\$ 1.427 por mujer con DM detectada por año. Esta cifra proviene del gasto anual en salud en Chile, de 21.200 millones de dólares norteamericanos, de los cuales, $10,2 \%$ fue destinado a $\mathrm{DM}^{27}$ Por lo tanto, el costo de detección y tratamiento de paciente nuevo es de US\$ 433+ US\$ $1.427=$ US\$ 1.860 por paciente detectada por año.

\section{Años de vida perdidos}

La Tabla 5 muestra la frecuencia de cada uno de los tipos y costos de malformaciones congénitas que afectan a embarazos chilenos, prorrateados tomando como base el número total de malformaciones anuales en Chile.

Calculamos tres tipos de cifras: los años de vida perdidos por muerte prematura (YLL), los años perdidos por discapacidad (YLD, en inglés) y los años de vida ajustados por discapacidad (DALY, en inglés). (i) Para ambos escenarios A y B, los YLL se calcularon como la suma del número incremental de infantes con malformaciones, mencionados en las Tablas 6 y 7. (ii) Revisamos la literatura para determinar, para cada uno de 29 tipos de malformación, los años de vida perdidos (Tabla 5). En la misma Tabla, la proporción de diferentes tipos de malformaciones en Chile fue prorrateada, lo que nos permitió calcular una carga promedio por discapacidad (DW, por la expresión en inglés disability weight) de $0,6181^{28}$. En la misma Tabla 5 obtuvimos una cifra media preliminar de 20 años de vida perdidos por cada recién nacido con malformaciones.

Tanto los YLL como los YLD fueron calculados con una tasa de descuento de $5 \%$, como sigue en las ecuaciones 2 y 3 respectivamente:

$$
Y L L=\frac{N}{0,05}\left(1-e^{-0,05 L}\right)
$$

Ecuación 2

En que: $\mathrm{N}=$ número de muertes prematuras. El denominador 0,05 es la tasa de descuento del $5 \%$. $\mathrm{L}=$ expectativa de vida a la edad de la muerte en años.

$$
Y L D=\frac{I D W\left(1-e^{-0,05 L}\right)}{0,05}
$$

Ecuación 3

En que: $\mathrm{I}=$ número de casos incidentes. DW $=$ carga de discapacidad ${ }^{25}$. $\mathrm{L}=$ duración media de la vida del paciente hasta la remisión de la enfermedad o la muerte (años).

$$
D A L Y=I L L+Y L D
$$

Ecuación 4 


\section{Resultados}

\section{Mujeres en edad fértil en 2017 y su proyección al año 2040}

En el año 2017, se registraron 4.496.190 MEF en Chile. La ecuación de regresión lineal entre tiempo transcurrido ( $x$, en años calendario) y población $\operatorname{MEF}(y)$, calculada para los datos 2003, 2010 y 2017 es la siguiente:

$$
y=8002 x-1,175 \times 10^{7}
$$

\section{Ecuación 5}

$\mathrm{Al}$ extrapolar la ecuación 5, las MEF en Chile llegarían a 4.574.080 en el año 2040.

\section{Prevalencia de diabetes mellitus en MEF en 2017 y su proyección a 2040}

En la Tabla 2 podemos ver que la prevalencia actual de MEF-DM es de 6,6\%. La Figura 2 muestra que las prevalencias de MEF-DM de Estados Unidos de Norteamérica (U.S.A. ${ }^{29}$ y de Chile convergerán en 16\% en el año 2040. De las 4.496.190 MEF que hay en Chile (2017), 296.834 $(6,6 \%)$ son MEF-DM (Tabla 3-MS). Al extrapolar una regresión lineal basada en las cifras entre 2003 y 2017, estimamos una cifra de 715.569 MEF-DM para el año 2040 (Figura 3). Con el fin de disponer de una sola cifra de prevalencia para el período 2020-2039, elegimos el número proyectado de MEF-DM a la mitad del período, año 2030, el cual, de acuerdo a la ecuación que se muestra en Figura 3, es de 537.100.

La Figura 4 muestra el diagrama de influencia para el modelo de Markov en el escenario A. La ecuación 6 muestra el vector de ciclo cero, arriba, multiplicando a la matriz $P$,

\section{$\left[\begin{array}{llllll}488.761 & 6.670 & 41.662 & 0,00000 & 0,00000 & 0,00000\end{array}\right]$ \\ $\left[\begin{array}{lllllll}0,91000 & 0,01242 & 0,07757 & 0,00000 & 0,00000 & 0,00000\end{array}\right.$ $\begin{array}{llllll}0,00000 & 0,00000 & 0,00000 & 0,00351 & 0,00000 & 0,99649\end{array}$ $\begin{array}{llllll}0,00000 & 0,00000 & 0,00000 & 0,00000 & 0,99649 & 0,00351\end{array}$ $\begin{array}{llllll}0,00000 & 0,00000 & 0,00000 & 1,00000 & 0,00000 & 0,00000\end{array}$ $\begin{array}{lllllll}0,00000 & 0,00000 & 0,00000 & 0,00000 & 1,00000 & 0,00000\end{array}$ $\left[\begin{array}{llllll}0,00000 & 0,00000 & 0,00000 & 0,00000 & 0,00000 & 1,00000\end{array}\right]$ \\ $=\left[\begin{array}{llllll}81448 & 1111 & 6942 & 216 & 384424 & 62905\end{array}\right]$}

Ecuación 6
De los seis estados finales ordenados de izquierda a derecha en el vector abajo en la ecuación 6 , la suma de dos veces el estado 4, más una vez el estado 6, resulta en un total de 63.337 niños con malformaciones que nacerían en los próximos 20 años si Chile sigue en el escenario A de no-intervención.

La Figura 5 muestra el diagrama de influencia para el modelo de Markov en el escenario B.

\section{$\left[\begin{array}{lllllll}488761 & 1885 & 46194 & 0,00000 & 0,00000 & 0,00000\end{array}\right]$ \\ $\left[\begin{array}{llllll}0,91000 & 0,00351 & 0,08649 & 0,00000 & 0,00000 & 0,00000 \\ 0,00000 & 0,00000 & 0,00000 & 0,00351 & 0,00000 & 0,99649 \\ 0,00000 & 0,00000 & 0,00000 & 0,00000 & 0,99649 & 0,00351 \\ 0,00000 & 0,00000 & 0,00000 & 1,00000 & 0,00000 & 0,00000 \\ 0,00000 & 0,00000 & 0,00000 & 0,00000 & 1,00000 & 0,00000 \\ 0,00000 & 0,00000 & 0,00000 & 0,00000 & 0,00000 & 1,00000\end{array}\right]$ \\ $=\left[\begin{array}{llllll}81448 & 314 & 7741 & 61 & 428371 & 18903\end{array}\right]$}

Ecuación 7

En el vector al final de la ecuación 7, la suma de dos veces el estado 4, más una vez el estado 6, resulta en un total de 19.025 niños con malformaciones que nacerían en los próximos 20 años si Chile aplica el escenario B de intervención.

En el escenario B, al aplicar los US\$ 1.860 por paciente detectada por año, con $5 \%$ de descuento, a las 252.007 MEF-DMnoDx, resulta en una cifra global de costo de detección y tratamiento de pacientes nuevas de US\$292.064.361, los que, al sumarse a los US\$3.062.257.607 de costo de por vida, dan un costo total para escenario B de US\$ 3.354.321.968 (Tabla 8).

\section{Discusión}

La hiperglicemia materna periconcepcional induce malformaciones fetales ${ }^{30}$ a través de estrés oxidativo, estrés por nitrosaminas, estrés de retículo endoplásmico, estrés por hexosamina y apoptosis ${ }^{7,9,10}$. Cuando en 2018 publicamos las cifras de malformaciones congénitas relacionadas con diabetes pregestacional esperadas para los siguientes 20 años, basamos nuestros cálculos en la Encuesta Nacional de Salud del año 2010. Sin embargo, al publicarse en 2019 los resultados preliminares de la ENS-2017, nos dimos cuenta que la prevalencia de DM en mujeres chilenas había aumentado nuevamente en todo el rango 15-49 

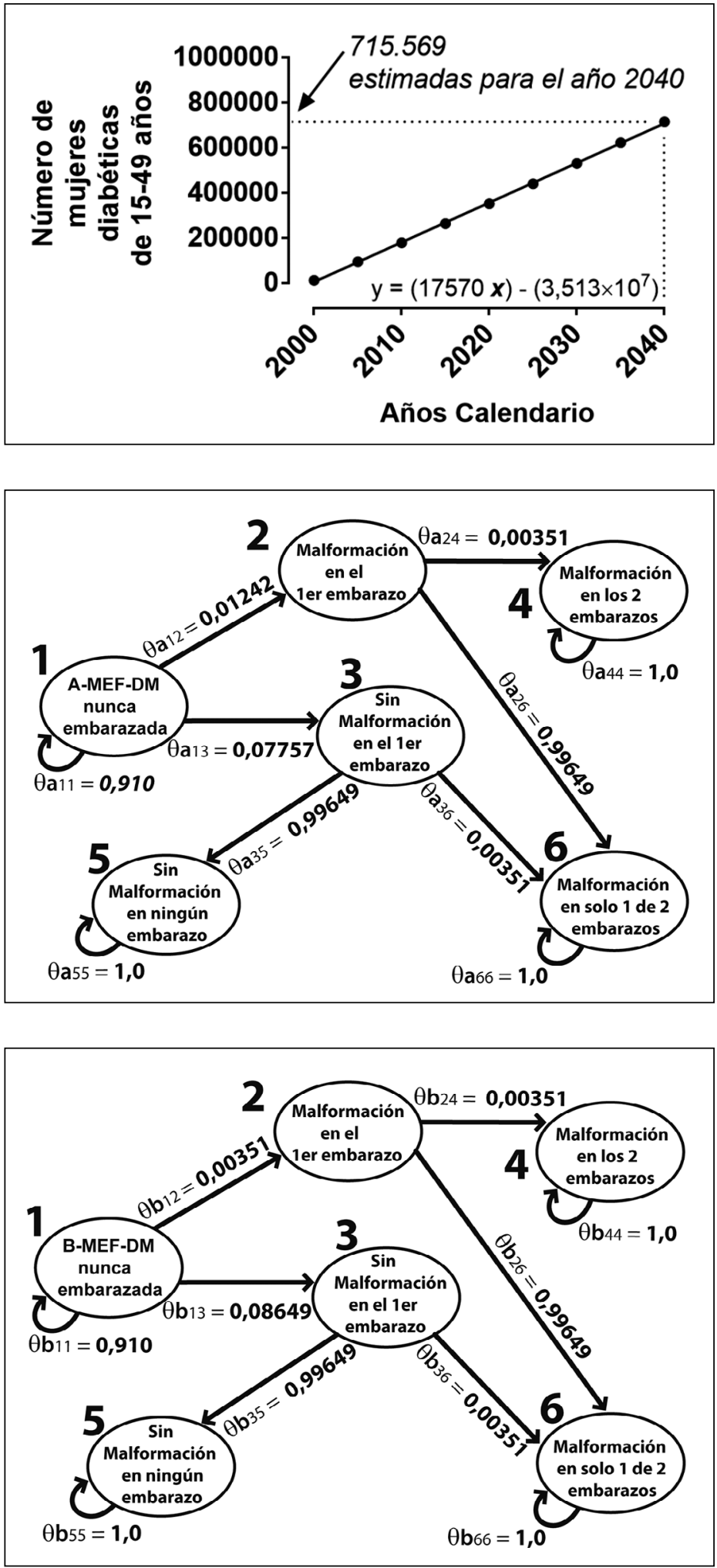

Figura 3. Regresión lineal de mujeres chilenas en edad fértil (15-49 años) con DM versus año calendario entre 2000 y 2040. Para el año 2040, se estima un número de 715.569 .

Figura 4. Diagrama de Influencia. Escenario " $A$ " (sin intervención): Malformaciones congénitas para el período 2020-2039, en mujeres chilenas en edad fértil (15-49 años) con DM. Cada uno de los 6 óvalos representa uno de los 6 estados. Las flechas con los símbolos $\left(\theta_{a 12}, \theta_{a 11}, \theta_{a 24}, \theta_{a 36}, \theta_{a 35}, \theta_{a 44}, \theta_{a 55}, \theta_{a 66}\right)$ representan las probabilidades de transición entre los estados. Observe que los estados 4 5 y 6 son absorbentes, de los cuales no hay salida, de modo que las probabilidades de permanecer en ellos $\left(\theta_{a 44}, \theta_{a 55}, \theta_{a 66}\right)$ son todas iguales a uno.

Figura 5. Diagrama de Influencia. Escenario " $B$ " (con intervención): Malformaciones congénitas para el período 2020-2039, en mujeres chilenas en edad fértil (15-49 años) con DM. Cada uno de los 6 óvalos representa uno de los 6 estados. Las flechas con los símbolos $\left(\theta_{\mathrm{b} 12}, \theta_{\mathrm{b} 11}, \theta_{\mathrm{b} 24}, \theta_{\mathrm{b} 36}, \theta_{\mathrm{b} 35}, \theta_{\mathrm{b} 44}, \theta_{\mathrm{b} 55}, \theta_{\mathrm{b} 66}\right)$ representan las probabilidades de transición entre los estados. Observe que los estados 4, 5 y 6 son absorbentes, de los cuales no hay salida, de modo que las probabilidades de permanecer en ellos $\left(\theta_{\mathrm{b} 44}, \theta_{\mathrm{b} 55}, \theta_{\mathrm{b} 66}\right)$ son todas iguales a uno. 
años, lo que hizo necesario y urgente actualizar nuestro modelo de Markov.

En efecto, no solo encontramos que el número proyectado de malformaciones (63.339 para los próximos 20 años) había subido 5,2 veces en relación a los cálculos basados en la ENS-2010, sino que los costos totales habían aumentado en esa misma magnitud, subiendo desde mil novecientos a casi diez mil doscientos millones de dólares norteamericanos.

Para el(la) lector(a) que compare este manuscrito con nuestra publicación de 2018 sobre el mismo tema ${ }^{13}$, puede parecer extraño, a primera vista, que en la primera publicación se usó 44 años como límite del rango de edad fértil, mientras que en el presente trabajo usamos el rango 15-49 años. Esto se debe a que en 2016, el MINSAL adoptó esta última definición ${ }^{31}$.

La introducción en Chile de un programa de detección de DM en mujeres de 15-49 años tendría el potencial de reducir la cifra de malformaciones y sus costos en cerca de $70 \%$. Lo que proponemos es que, cada vez que una mujer en edad fértil consulte a un obstetra-ginecólogo o a una matrona, por cualquier razón, se le indique un examen de hemoglobina glicosilada, lo que tiene dos ventajas: (a) No requiere que la paciente esté en ayunas, $y$ (b) su resultado, aparte de determinar si la paciente debe ser investigada más exhaustivamente, entrega simultáneamente un riesgo de malformaciones congénitas.

Uno de los parámetros de nuestro modelo, la incidencia de malformaciones congénitas, ya no se podrá considerar como constante en el futuro, porque en el período 2010-2019 se han publicado dos trabajos que sugieren que esta puede estar aumentando. En la Región del Maule, la incidencia de malformaciones subió de 1,66\% en 2012, a $2,01 \%$ en $2014^{32}$, y en la Región de Los Lagos, esta subió de $2,7 \%$ en 2011 a 9,06\% en $2017^{33}$. Estas cifras son regionales y no nacionales, ya que a la fecha, Chile no está entre los 11 países de Latinoamérica y Caribe que tienen registros gubernamentales de malformaciones congénitas ${ }^{34,35}$. Hasta 2014, las cifras de nuestro país han sido publicadas por $\mathrm{Nazer}^{22}$, gracias a la meritoria asociación de él y otros autores con el Estudio Colaborativo Latinoamericano de Malformaciones Congénitas (ECLAMC), fundado en 1967.

La principal fortaleza de nuestro modelo es que identificamos los posibles efectos de la creciente prevalencia de DMT2 en mujeres jóvenes en Chile, antes que ocurra un alza significativa de malformaciones congénitas. Este enfoque preventivo es, en nuestra opinión, mucho más efectivo que una respuesta reactiva cuando el problema alcance proporciones epidémicas. Su costo, por otra parte, es menor que lo que, a veces, se pretende invertir en enfermedades infrecuentes que requieren tratamientos extremadamente caros.

Una de las debilidades de nuestra investigación es que, para los próximos 20 años, introducimos el concepto de "la cifra media de 537.100 mujeres en edad fértil con DMT2". Podemos decir en nuestro favor que esto fue necesario dado el continuo aumento en la prevalencia de DM en mujeres en edad fértil, lo que nos obligó a introducir la media aritmética entre la prevalencia actual (296.834) y la cifra proyectada para el año 2040 (715.569). La cifra media mencionada es la proyectada para el año 2030, vale decir, 537.100 MEF-DM.

Otra debilidad de nuestra investigación es que, debido a las diferencias en los rangos de edad publicados por el Ministerio de Salud por un lado, y por el Instituto Nacional de Estadísticas, por otro, la prevalencia de DM publicada para el rango-MINSAL "45-64" (18,3\%) tuvimos que aplicarla a la población en el rango-INE “45-49”.

Nuestro modelo adolece de no haber tenido en cuenta que en el período etario en que la fertilidad es mayor, la prevalencia de DM es menor y en el grupo de mayor edad, la fertilidad es mucho menor y la prevalencia de diabetes mellitus es mayor.

Sin embargo, la principal debilidad consiste en que en el escenario B estamos asumiendo que, una vez diagnosticada la DMT2, 100\% de las mujeres ingresaría en un estricto protocolo de tratamiento, capaz de mantener su HbAlc por debajo de 6,5\%. A primera vista, esta sería una suposición ingenua, pero lo que de hecho intentamos hacer fue aplicar el enfoque matemático reductio ad absurdum, asumiendo que en la mejor situación concebible del escenario B, todas las MEF-DM en Chile tendrían su DMT2 diagnosticada y tratada eficazmente.

Teniendo en cuenta estas limitaciones, y en el contexto de la epidemia mundial de diabetes mellitus ${ }^{36}$, nuestra línea de investigación, en su fase actual de modelo matemático de Markov, constituye un primer paso para evitar un aumento catastrófico de nacimientos con malformaciones congénitas relacionados con la DM no solo en Chile, sino en todo el mundo. 


\section{Referencias}

1. Kaempffer AM, Medina E. Mortalidad infantil reciente en Chile: éxitos y desafíos. Rev Chil Pediatr 2006; 77: 492-500. http://dx.doi.org/10.4067/S037041062006000500007.

2. Atrash HK, Johnson K, Adams M, Cordero JF, Howse J. Preconception Care for Improving Perinatal Outcomes: The Time to Act. Matern Child Health J 2006; 10 (Suppl 1): 3-11. https://dx.doi.org/10.1007\%2Fs10995-006-0100-4.

3. Peterson C, Grosse SD, Li R, Sharma AJ, Razzaghi H, Herman WH, Gilboa SM. Preventable health and cost burden of adverse birth outcomes associated with pregestational diabetes in the United States. Am J Obstet Gynecol 2015; 212 (1): 74.e1-e9. https://doi. org/10.1016/j.ajog.2014.09.009.

4. Yehuda I. Implementation of Preconception Care for Women with Diabetes. Diabetes Spectr 2016; 29 (2): 105-14. https://dx.doi.org/10.2337\%2Fdiaspect.29.2.105.

5. Wilmot E, Idris I. Early onset type 2 diabetes: risk factors, clinical impact and management. Ther Adv Chronic Dis 2014; 5 (6) 234-44. https://dx.doi.org/10.1177\%2F2040622314548679.

6. Cundy T, Gamble G, Townend K, Henley PG, Mac-Pherson P, Roberts AB. Perinatal mortality in Type 2 diabetes mellitus. Diabet Med 2000; 17 (1): 33-9.

7. Olmos P, Belmar C. Diabetes and pregnancy. In: A Pérez-Sánchez A, Donoso Siña E (editores). Obstetricia, $4^{\circ}$ edición. Santiago, Chile: Editorial Mediterráneo, 2011; páginas 1019-1050. ISBN: 978-956-220-314-2.

8. Guerin A, Nisembaum R, Ray JG. Use of maternal GHb concentration to estimate the risk of congenital anomalies in the offspring of women with pregestational diabetes. Diabetes Care 2007; 30: 1920-5. https://doi. org/10.2337/dc07-0278.

9. Gestation and Diabetes in France Study Group. Multi-centric survey of diabetic pregnancy in France. Diabetes Care 1991; 14: 994-1000. https://doi.org/10.2337/ diacare.14.11.994.

10. Jensen DM, Damm P, Moelsted-Pedersen L, Ovesen P, Westgaard JG, Moeller M, et al. Outcomes in type 1 diabetes pregnancies. A nationwide, population-based study. Diabetes Care 2004; 27 (12): 2819-23. https://doi. org/10.2337/diacare.27.12.2819.

11. Gabbay-Benziv R, Reece EA, Wang F, Yang P. Birth defects in pregestational diabetes: Defect range, glycemic threshold and pathogenesis. World J Diabetes. 2015; 6 (3): 481-8. https://dx.doi.org/10.4239\%2Fwjd.v6.i3.481.

12. Fagot-Campagna A, Pettitt DJ, Engelgau MM, Burrows
NR, Geiss LS, Valdez R, et al. Type 2 diabetes among North adolescents: An epidemiologic health perspective. J Pediatr 2000; 136: 664-72. https://doi.org/10.1067/ mpd.2000.105141.

13. Olmos P, Borzone G, Poblete A. 47-Fold rise of diabetes in childbearing age Chilean women: Markov model and cost-effectiveness of prevention of birth defects. J Obstet Gynaecol Res 2018; 44 (9): 1719-30. https://doi. org/10.1111/jog.13712.

14. MINSAL-Chile (2017). Ministerio de Salud, Chile. Departamento de Epidemiología División de Planificación Sanitaria Subsecretaría de Salud Pública. Tabla: Sospecha de Diabetes Mellitus según grupo de edad. ENS 2009-10 y 2016-17. ENCUESTA NACIONAL DE SALUD 2016-2017 Primeros resultados. https://www. minsal.cl/wp-content/uploads/2017/11/ENS-201617_PRIMEROS-RESULTADOS.pdf (Consultado el 26 de agosto de 2019).

15. MINSAL-Chile (2003). Ministerio de Salud, Chile. Tabla IV.4.8.- Prevalencia de diabetes por edad y sexo. Encuesta Nacional de Salud Chile 2003. Página 197. http://epi.minsal.cl/wp-content/uploads/2016/03/InformeFinalENS2003.vent_.pdf (Consultado el 26 de agosto de 2019).

16. INE-Chile (2003). Instituto Nacional de Estadísticas. Comisión Nacional Chilena del XVII Censo de Población y Vivienda. Resumen de Resultados. Características Demográficas. Página 13. https://www.ine.cl/docs/default-source/FAQ/s\%C3\%ADntesis-de-resultados-censo-2002. pdf?sfvrsn=2 (Consultado el 2 de agosto de 2019).

17. PNUD-Chile (2013). Informe final. Comisión externa revisora del Censo de Chile del año 2012. http://www. cl.undp.org/content/chile/es/home/library/poverty/ informes_de_comisiones/informe-final--comision-externa-revisora-del-censo-2012.html (Consultado el 7 de agosto de 2019).

18. INE-Chile (2017). Instituto Nacional de Estadísticas (2017). Resultados del Censo 2017. http://resultados. censo2017.cl/ (Consultado el 7 de agosto de 2019).

19. MINSAL-Chile (2010a). Ministerio de Salud, Chile. Tabla III.1.3.1. Distribución de la población chilena de 15 y más años, según edad y sexo (Resumido de: Censo de Población y Viviendas, INE, abril de 2002). En: Sección III, Metodología. En: Encuesta Nacional de Salud Chile 2009-2010. Página 6. https://www.minsal.cl/portal/url/ item/bcb03d7bc28b64dfe040010165012d23.pdf (Consultado el 7 de agosto de 2019).

20. MINSAL-Chile 2010b. Tabla V.2.4.10. Conocimiento, tratamiento y control de la diabetes por sexo.Ministerio de Salud-Chile. Encuesta Nacional de Salud 2009-2010. Página 149 [Consultado el 13 de agosto de 2019] Dis- 
ponible en: http://www.minsal.gob.cl/portal/url/item/ bcb03d7bc28b64dfe040010165012d23.pdf.

21. The World Bank Group (2019). Fertility rate, total (births per woman). https://data.worldbank.org/indicator/ sp.dyn.tfrt.in?end=2017\&start $=1960$ (Consultado el 2 de agosto de 2019).

22. Nazer J, Cifuentes L. [Prevalence of congenital malformations at birth in Chilean maternity hospitals]. Rev Med Chile 2014; 142: 1150-6. http://www.scielo.cl/pdf/ rmc/v142n9/art09.pdf.

23. Drummond MF, Sculpher MJ, Torrance GW, O’Brien BJ, Stoddart GL. En: Chapter 9: Economic evaluation using decision analytic modelling. En: Drummond MF, Sculpher MJ, Torrance GW, O'Brien BJ, Stoddart GL (editores). Methods of the Economic Evaluation of Health Care Programmes, 3rd edition. Oxford: Oxford University Press, 2005; 277-322.

24. Gillett M, Brennan A, Watson P, Khunti K, Davies $\mathrm{M}$, Mostafa S, et al. The cost-effectiveness of testing strategies for type-2 diabetes: a modelling study. Health Technology Assessment 2015; 19 (33): 45-6. ncbi.nlm. nih.gov/books/NBK293799/pdf/Bookshelf_NBK293799. pdf.

25. Whitley HP, Yong EV, Resinen C. Selecting an A1C point-of care instrument. Diabetes Spectr 2015; 28: 2018. https://doi.org/10.2337/diaspect.28.3.201.

26. Whitley HP, Hanson C, Parton JM. Systematic diabetes screening using point-of-care hbalc testing facilitates identification of prediabetes. Ann Fam Med 2017; 15: 162-4. https://doi.org/10.1370/afm.2035.

27. Sapunar J. Chilean epidemiology in diabetes mellitus. [Rev Med Clin Condes] 2016; 27: 146-51. https://doi. org/10.1016/j.rmclc.2016.04.003.

28. World Health Organization. The Global Burden of Disease: 2004 Update. In: Disability Weights for Diseases and Conditions. Geneva: World Health Organization, 2004. Disponible en: http://www.who.int/healthinfo/ global_burden_disease/GBD2004_DisabilityWeights. pdf (Consultado el 26 de julio de 2019).

29. Ginter E, Simko V (2013). Prevalence and forecast in percent population in USA, diagnosed to be suffering from diabetes by age-group and selected years. In: Chapter 5, Global prevalence and future of diabetes mellitus. In: Ahmad SI, Editor. Diabetes An Old Disease, a New Insight. 2013 by Landes Bioscience and Springer Science+Business Media, LLC, Rio Grande, Austin, Texas 78701, USA. Page 40. https://link.springer.com/content/ pdf/10.1007\%2F978-1-4614-5441-0.pdf (Consultado el 7 de agosto de 2019).

30. Guerin A, Nisenbaum R, Ray JG. Use of maternal GHb concentration to estimate the risk of congenital anoma- lies in the offspring of women with pregestational diabetes. Diab Care 2007; 30: 1920-5. https://doi.org/10.2337/ dc07-0278.

31. Ministerio de Salud de Chile (MINSAL), Programa Nacional Salud de la Mujer Instituto Chileno de Medicina Reproductiva (ICMER) Asociación Chilena de Protección de la Familia (APROFA), 2016. Normas Nacionales Sobre Regulación De La Fertilidad Ministerio de Salud de Chile. ISBN versión PDF: 978956-348-151-8. https://www.minsal.cl/wp-content/ uploads/2015/09/2018.01.30_NORMAS-REGULACION-DE-LA-FERTILIDAD.pdf (Consultado el 7 de agosto de 2019).

32. Widerström J, Ugarte D, Cortés J, Arratia A, Plaza B, Pohlhammer B. Prevalencia de malformaciones congénitas en recién nacidos de la Región del Maule entre los años 2009 al 2014. Medwave 2016; 16 (Suppl 6): e6666. https://doi:10.5867/medwave.2016.6666. https://www. medwave.cl/link.cgi/Medwave/ResumenesCongreso/ sp2016/EstatEnSalud/6666.act (Consultado el 30 de enero de 2020).

33. Maldonado C, Murer A, Flores L, Rojas M, Conei D. Prevalencia de malformaciones congénitas en la Región de Los Lagos, Chile, período 2015-2017. J Health Med Sci 2019; 5 (4): 261-7. http://www.johamsc.com/ wp-content/uploads/2019/12/JOHAMSC-54-261-2672019.pdf.

34. Durán P, Liascovich R, Barbero P, et al. Sistemas de vigilancia de anomalías congénitas en América Latina y el Caribe: presente y futuro. Rev Panam Salud Publica 2019; 43: e44. https://doi:10.26633/RPSP.2019.44.

35. PAHO. Pan American Health Organization. Birth defects registries expanding in Latin America. PAHO, Montevideo, 8 de agosto de 2019. https://www.paho. org/hq/index.php?option $=$ com_content\&view $=$ article\&id=15352:birth-defects-registries-expanding-in-latin-america\&Itemid $=1926 \&$ lang $=$ en (Consultado el 30 de enero de 2020).

36. Zarante I, Hurtado-Villa P, Walani SR, Kancherla V, López Camelo J, Giugliani R, et al. A consensus statement on birth defects surveillance, prevention, and care in Latin America and the Caribbean. Rev Panam Salud Pública. 2019; 43:e2. https://doi.org/10.26633/ RPSP.2019.2.

37. World Health Organization. Global Report on Diabetes. 2016 by WHO Press, World Health Organization, 20 Avenue Appia, 1211 Geneva 27, Switzerland. Pp.6. https://apps.who.int/iris/bitstream/handle/10665/204871/9789241565257_eng.pdf;jsessionid=CB1F3C0E22DA4E8DEBA03FD56FD81115? sequence $=1$ (Consultado el 26 de julio de 2019). 
38. Wynbrandt J, Ludman MD. Appendix II. Average lifetime costs per child with selected birth defects, 2001. In: Wynbrandt J, Ludman MD. Encyclopedia of Genetic Disorders and Birth Defects. Facts On File, Inc. An inprint (2008) of Infobase Publishing, 132 West 31th Street, New York, NY 10001, USA. Page 514. https://books. google.cl/books?isbn=1438120958 (Consultado el 17 de octubre de 2016).

39. Goic A. [The Chilean Health Care System: The task ahead]. Rev Med Chile 2015; 143: 774-86. http://www. scielo.cl/pdf/rmc/v143n6/art11.pdf.

40. NDSS. National Down Syndrome Society. Down Syndrome Fact Sheet. http://www.ndss.org/Down-Syndrome/Down -Syndrome-Facts/ (Consultado el 20 de mayo de 2019).

41. Rajasinghe HA, McElhinney DB, Reddy VM, Mora BN, Hanley FL. Long-term follow-up of truncus arteriosus repaired in infancy: a twenty-year experience. J Thorac Cardiovasc Surg 1997; 113 (5): 869-78. https://doi. org/10.1016/S0022-5223(97)70259-9.

42. U.S. Department of Health, Education and Welfare. Public Health Service. Table 5-1. Abridged Life Tables by Color and Sex: United States, 1975. In: Section 5, Life Tables. In: Volume II, Vital Statistics of the United States 1975. Page 5.8. www.cdc.gov/nchs/data/lifetables/ life75.pdf (Consultado el 20 de mayo de 2019).

43. Görler H, Ono M, Thies A, Lunkewitz E, Westhoff-Bleck $\mathrm{M}$, Haverich A, et al. Long-term morbidity and quality of life after surgical repair of transposition of the great arteries: atrial versus arterial switch operation. Interact Cardiovasc Thorac Surg 2011; 12 (4): 569-74. https:// doi.org/10.1510/icvts.2010.253898.

44. Nollert G, Fischlein T, Bouterwek S, Böhmer C, Klinner W, Reichart B. Long-term survival in patients with repair of tetralogy of Fallot: 36-year follow-up of 490 survivors of the first year after surgical repair. J Am Coll Cardiol. 1997; 30 (5): 1374-83. https://doi.org/10.1016/ s0735-1097(97)00318-5.

45. Clínica Alemana (Santiago, Chile). Institutional Price List. Clínica Alemana, Santiago, Chile, 2014. http:// www.alemana.cl/amca/ArancelAmca.pdf (Consultado el 20 de agosto de 2019).

46. Strauss D. Life Expectancy Project, San Francisco, CA, USA. Evidence-Based Life Expectancy. Dev Med Child Neurol 2010; 52 (8): 695. https://doi.org/10.1111/j.14698749.2009.03563.x.

47. Oakeshott P, Hunt GM, Poulton A, Reid F. Expectation of life and unexpected death in open spina bifida: a 40-year complete, non-selective, longitudinal cohort study. Dev Med Child Neurol 2010 Aug; 52 (8): 749-53. https://doi.org/10.1111/j.1469-8749.2009.03543.x
48. Siffel C, Wong LY, Olney RS, Correa A. Survival of infants diagnosed with encephalocele in Atlanta, 197998. Paediatr Perinat Epidemiol 2003 Jan; 17 (1): 40-8. https://doi.org/10.1046/j.1365-3016.2003.00471.x.

49. Pérez JC. Table 11: Abbreviated table of mortality for both sexes. 2001-2002. In: CHILE: Tablas Abreviadas de Mortalidad por Sexo Total País y Regiones. 20012002 INSTITUTO NACIONAL DE ESTADÍSTICAS. Santiago, Chile, 2004. Página 29. http://www.ine.cl/ canales/chile_estadistico/demografia_y_vitales/estadisticas_vitales/pdf/tablasmortalidadporsexo2001_02.pdf (Consultado el 30 de mayo de 2019).

50. BINOCAR. British Isles Network of Congenital Anomaly Registers. Congenital Anomaly Statistics 2012 England and Wales. Table 7.5. Page 47. http://www. binocar.org/content/Annual\%20report\%202012_FINAL_nologo.pdf (Consultado el 30 de julio de 2019).

51. RIM A, Orth MM, Mohd-Hisyamudin HP, Ahmad $\mathrm{MH}$, Zulkiflee O, Orth MS. The Economic Impact of Managing Late Presentation of Developmental Dysplasia of Hip (DDH). Malaysian Orthopaedic Journal 2015; 9 (3): 40-3. https://dx.doi.org/10.5704\%2FMOJ.1511.006

52. Steinhorn RH, Kriesmer PJ, Green TP, McKay CJ, Payne NR. Congenital diaphragmatic hernia in Minnesota. Impact of antenatal diagnosis on survival. Arch Pediatr Adolesc Med 1994; 148 (6): 626-31. https://doi. org/10.1001/archpedi.1994.02170060080016.

53. Henrich K, Huemmer HP, Reingruber B, Weber PG. Gastroschisis and omphalocele: treatments and longterm outcomes. Pediatr Surg Int 2008; 24 (2): 167-73. https://doi.org/10.1007/s00383-007-2055-y

54. Clark DC. Esophageal Atresia and Tracheoesophageal Fistula. Am Fam Physician 1999; 59 (4): 910-6. http:// www.aafp.org/afp/1999/0215/p910.html (Consultado el 10 de enero de 2017).

55. Singal AK. Cost of Hypospadias Surgery in India. Hypospadias Foundation. http://www.hypospadiasfoundation.com/hypospadias-blog-by-dr-a-k-singal/cost-ofhypospadias-surgery-in-mumbai-india/ (Consultado el 20 de agosto de 2019).

56. Bell JC, Nassar N, Bower C, Turner RM, Raynes-Greenow C. Long-term survival for infants born with orofacial clefts in Western Australia. Birth Defects Res A Clin Mol Teratol 2016; 106 (3): 172-7. https://doi. org/10.1002/bdra.23473.

57. Warf BC. Statement of Benjamin C. Warf Director, Neonatal and Congenital Anomalies Neurosurgery Children's Hospital Boston Associate Professor of Surgery Harvard Medical School August 2, 2011 House Committee on Foreign Affairs, Subcommittee on Africa, Global Health, and Human Rights. http://archives. 
republicans.foreignaffairs.house.gov/112/war080211.pdf (Consultado el 20 de agosto de 2019).

58. Academic Pediatric Association plus 69 other Societies of the U.S.A. Zika-coalition-House-Senate-letters. Testimony to the U.S. Congress, April 5th, 2016. http:// www.aafp.org/dam/AAFP/documents/news/Zika-coalition-House-Senate-letters.pdf (Consultado el 23 de agosto de 2019).

59. Eriksson D, Karlsson L, Eklund O, Dieperink H, Honkanen E, Melin J, et al. Autosomal Polycystic Kidney Disease - Costs and Resource Utilisation in the Nordic Countries. Value Health 2015; 18 (7): A513. https://doi. org/10.1016/j.jval.2015.09.1487.

60. Millar AJW, Rode H, Cywes S. Intestinal atresia and stenosis. En: Ashcraft KW, Holder TM, eds. Pediatric Surgery, New York, USA, Saunders, 2000, páginas 40624.

61. Hsieh MH, Meng MV, Baskin LS. Outcomes and cost analysis of pyeloplasty for antenatally diagnosed ureteropelvic junction obstruction using Markov models. NHS Economic Evaluation Database (NHS EED) Produced by the Centre for Reviews and Dissemination Copyright $\odot 2016$ University of York, UK. http:// www.crd.york.ac.uk/crdweb/ShowRecord.asp?LinkFrom=OAI\&ID=22008102131 (Consultado el 23 de agosto de 2019).

62. Pomerance JJ, Morrison A, Williams RL, Schifrin BS. Anencephalic infants: life expectancy and organ donation. J Perinatol 1989; 9 (1):33-7.

63. Castilla I, Vallejo-Torres L, Rica-Echevarría I, Rodríguez-Sánchez A, Dulín-Íñiguez E, Espada $\mathrm{M}$, et al. Cost-effectivity of neonatal screening of congenital adrenal hiperplasia. Ministry of Health, Social Services and Equality. Evaluation Service of the Canarian (Spain) Health Service: 2013. Evaluation report of health technologies. Table $\mathrm{N}^{\circ}$ 14. Page 50. http://www3. gobiernodecanarias.org/sanidad/scs/content/8a194cda-f3ed-11e4-aa6f-cfdf8d9a72f8/SESCS\%202013_C\%20 N\%20H.Suprarrenal.pdf (Consultado el 23 de agosto de 2019).

64. Costa RN, Ribeiro MS, Ferreira da Silva A, Ribeiro RA, Berwanger O, Biasi A, et al. Incremental Cost-Effectiveness of Surgical vs. Percutaneous Treatment of Patent Ductus Arteriosus with the Amplatzer ${ }^{\mathrm{TM}}$ Duct Occluder in Children: A Systematic Review. Rev Bras Cardiol Invasiva 2014; 22 (2): 168-79. http://dx.doi. org/10.1590/0104-1843000000029.

65. Shetty S, Kennea N, Desai P, Giuliani S, Richards J. Length of stay and cost analysis of neonates undergoing surgery at a tertiary neonatal unit in England. Ann R Coll Surg Engl 2016; 98: 56-60. https://doi.org/10.1308/ rcsann.2016.0034.

66. Paschoal-Nogueira M, Rodrigues Pereira JC, Duarte PS, Lourenco A, Tedesco AP, Alves-Ferreira L, et al. Brasil: Comparison of hospital costs and duration of treatment with two different clubfoot protocols. Iowa Orthop J. 2011; 31: 49-51. https://www.ncbi.nlm.nih.gov/pmc/ articles/PMC3215112/

67. Epidemiology \& Disease Control Division, Ministry of Health, Singapore. Singapore Burden of Disease Study 2010 ISBN 978-981-07-9564-1 June 2014. Page 62. https://www.moh.gov.sg/content/dam/moh_web/ Publications/Reports/2014/Singapore\%20Burden\%20 of\%20Disease\%20Study\%202010\%20Report_v3.pdf (Consultado el 23 de agosto de 2019).

68. Olmos P, Borzone G, Poblete A. 47-Fold rise of diabetes in childbearing age Chilean women: Markov model and cost-effectiveness of prevention of birth defects. J Obstet Gynaecol Res 2018; 44 (9): 1719-30. https://doi. org/10.1111/jog.13712. 